\title{
SOME PROPERTIES OF SOLUTIONS OF INITIAL-VALUE PROBLEMS ASSOCIATED WITH THE WAVE EQUATION*
}

\author{
BY \\ LI. (i. CHAMBERS \\ Iniveisity ('ollege of North Wales, Bangor, Wales
}

Summary. A variational principle and a reciprocity relation are obtained for initial value problems associated with the wave equation.

1. Introduction. (iurtin [1], [2] hats discussel this application of the convolution to the solution of initial-value problems. He discussed the solution of the wave equation, but not the mixed boundary condition. Ainola [3], following Gurtin, obtained a reciprocity relation involving the solution of an initial-value problem for the Schrödinger wave equation. In this paper the variational principle for the mixed boundary condition for the wave equation and reciprocity relationships associated with initial value problems for the wave equation will be obtained.

2. Formulation of problem. I.et $\phi$ be the solution of the ware equation

$$
\nabla^{2} \phi-\frac{1}{c^{2}} \frac{\partial^{2} \phi}{\partial t^{2}}=f(\mathbf{x}, t)
$$

(The number of space dimensions $n$ is irrelevant; $\mathbf{x}$ represents the set $\left(\mathbf{x}_{1}, \cdots, \mathbf{x}_{n}\right)$. The set of values of $\phi$ and $\partial \phi / \partial t$ at $t=0$ are given by $\phi_{0}(\mathbf{x})$ and $\phi_{1}(\mathbf{x})$, respectively. The region under consideration is surrounded by a boundary $B$ on which

$$
\alpha \phi+\beta(\partial \phi / \partial n)=g
$$

where $\alpha, \beta$, and $g$ are all functions of position on the boundary $B . g$ may be a function of time as well. $B$ is divided into two portions, $B_{1}$ and $B_{2}$. (On $B_{1}, \beta$ is nonzero; on $B_{2}, \beta$ is zero.

The convolution integral is defined as follows. If $u(t), v(t)$ are functions of $t$,

$$
u * v \equiv \int_{0}^{t} u(t-s) v(s) d s .
$$

The conditions imposed on $u$ and $v$ have been discussed, for example, by Jones [4]. In particular

$$
1 * v=\int_{0}^{t} 1 v(s) d s=\int_{0}^{t} v(s) d s .
$$

It is well known that the convolution obeys the commutative and associative laws.

Fatation (2.1) may be written as

$$
\partial^{2} \phi / \partial t^{2}=c^{2}\left(\nabla^{2} \phi-f\right)
$$

* Received September 20, 1968; revised version received April 7, 1969. 
Integrating with respect to time,

$$
\partial \phi / \partial t=c^{2}\left[1 *\left(\nabla^{2} \phi-f\right)\right]+\phi_{1}(\mathbf{x}) .
$$

Integrating further with respect to time,

$$
\begin{aligned}
\phi & =c^{2}\left[1 * 1 *\left(\nabla^{2} \phi-f\right)\right]+t \phi_{1}(\mathbf{x})+\phi_{0}(\mathbf{x}) \\
& =c^{2}\left[1 * 1 * \nabla^{2} \phi\right]-h,
\end{aligned}
$$

where

$$
h=c^{2}[1 * 1 * f]-t \phi_{1}(\mathbf{x})-\phi_{0}(\mathbf{x}) .
$$

This form is slightly different from that given by Gurtin [2], but is more convenient for the subsequent analysis.

3. The variational principle. Consider the functional

$$
\begin{aligned}
F[\phi]= & \frac{1}{2} \int\left[\phi * \phi+c^{2}\left(1 * 1 * \nabla \phi^{*} \nabla \phi\right)\right] d \tau+\int \phi * h d \tau \\
& +\frac{c^{2}}{2} \int_{B_{1}} \frac{\alpha}{\beta}[1 * 1 * \phi * \phi] d \sigma-c^{2} \int_{B_{1}} \frac{[1 * 1 * g * \phi] d \sigma}{\beta} .
\end{aligned}
$$

$d \tau$ is an element of the region, $d \sigma$ is an element of the boundary, and

$$
\nabla \phi^{*} \nabla \psi=\sum_{n=1}^{n} \frac{\partial \phi}{\partial \mathbf{x}_{p}} * \frac{\partial \psi}{\partial \mathbf{x}_{p}} .
$$

Following the usual ideas of the calculus of variations, and assuming that everything is sufficiently continuous,

Now

$$
\begin{aligned}
\delta F= & \int\left[\phi * \delta \phi+c^{2}(1 * 1 * \nabla \phi * \nabla \delta \phi)+\delta \phi * h\right] d \tau \\
& +c^{2} \int_{B_{1}} \frac{1}{\beta}[1 * 1 *(\phi \alpha-g)] * \delta \phi d \sigma .
\end{aligned}
$$

Thus

$$
\begin{aligned}
\int(1 * 1 * \nabla \phi * \nabla(\delta \phi)) d \tau & =\int 1 * 1 *\left\{\nabla \cdot(\delta \phi * \nabla \phi)-\delta \phi * \nabla^{2} \phi\right\} d \tau \\
& =\int_{B}\left(1 * 1 * \delta \phi * \frac{\partial \phi}{\partial n}\right) d \sigma-\int\left(1 * 1 * \nabla^{2} \phi * \delta \phi\right) d \tau .
\end{aligned}
$$

$$
\begin{aligned}
\delta F= & \int \delta \phi *\left[\phi-c^{2}\left(1 * 1 * \nabla^{2} \phi\right)+h\right] d \tau \\
& +\int_{B} \delta \phi *\left[1 * 1 * \frac{\partial \phi}{\partial n}\right] d \sigma+\int_{B_{1}} \delta \phi *\left[1 * 1 * \frac{1}{\beta}(\alpha \phi-g)\right] d \sigma,
\end{aligned}
$$

using the associative and commutative properties. This can finally be written as

$$
\begin{aligned}
\hat{\delta} F= & \int \delta \phi *\left[\phi-c^{2}\left(1 * 1 * \nabla^{2} \phi\right)+h\right] d \tau \\
& +\int_{B_{2}} \delta \phi * 1 * 1 *\left[\alpha \phi+\beta \frac{\partial \phi}{\partial n}-g\right] \frac{d \sigma}{\beta}+\int_{B_{2}} \delta \phi * 1 * 1 * \frac{\partial \phi}{\partial n} d \sigma .
\end{aligned}
$$


There are now two cases to consider. If $B_{2}$ does not exist, that is, $\beta$ is nonzero everywhere on the boundary, $\delta F$ vanishes if

$$
\phi-c^{2}\left(1 * 1 * \nabla^{2} \phi\right)+h=0
$$

and if

$$
\alpha \phi+\beta \frac{\partial \phi}{\partial n}=g
$$

this holds irrespective of the actual value of $\delta \phi$.

Alternatively, if $\delta F$ vanishes, (2.2) and (2.7) follow because $\delta \phi$, although small, is arbitrary. (2.2) is in fact a natural boundary condition as no constraint is placed on $\delta \phi$ on the boundary.

If, however, $B_{2}$ exists, that is $\beta$ vanishes at some part of the boundary, and the boundary condition thus specifies $\phi$ only, it is necessary to impose the condition $\delta \phi=0$ on $B_{2}$. It may be remarked that it is always possible to consider the problem with $g$ zero for the following reason. We may let $\phi=\theta+\psi$ where $\theta(\mathbf{x}, t)$ is a function such that

$$
\begin{gathered}
\theta(\mathbf{x}, 0)=\phi_{0}, \quad\left[\frac{\partial}{\partial t} \theta(\mathbf{x}, t)\right]_{t=0}=\phi_{1}, \\
\alpha \theta+\beta \frac{\partial \theta}{\partial n}=g \text { on } B,
\end{gathered}
$$

and

$$
\nabla^{2} \theta-\frac{1}{c^{2}} \frac{\partial^{2} \theta}{\partial t^{2}}=0
$$

$\psi(\mathbf{x}, t)$ is a function such that

$$
\begin{gathered}
\psi(\mathbf{x}, 0)=0, \quad\left[\frac{\partial}{\partial t} \psi(\mathbf{x}, t)\right]_{t=0}=0, \\
\alpha \psi+\beta \frac{\partial \psi}{\partial n}=0 \text { on } B
\end{gathered}
$$

and

$$
\nabla^{2} \psi-\frac{1}{c^{2}} \frac{\partial^{2} \psi}{\partial t^{2}}=f
$$

If this is done, the discontinuities due to boundary data which are propagated on characteristic surfaces all appear in $\theta . \theta$ is determined by the usual processes, and the problem has been reduced to one for which $g$ is zero.

4. Reciprocity Theorem. Let $\psi_{1}$ be the solution of (2.7) associated with a source $p_{1}$ and $\psi_{2}$ that associated with a source $p_{2}$. In both cases the boundary condition is (2.2).

It is not difficult to see that

$$
\begin{aligned}
\int_{B}\left[\psi_{1} * 1 * 1 * \frac{\partial \psi_{2}}{\partial n}-\psi_{2}\right. & \left.* 1 * 1 * \frac{\partial \psi_{1}}{\partial n}\right] d \sigma \\
& =\int\left[\psi_{1} * 1 * 1 * \nabla^{2} \psi_{2}-\psi_{2} * 1 * 1 * \nabla^{2} \psi_{1}\right] d \tau,
\end{aligned}
$$


this being a generalization of Green's theorem. From the boundary condition,

$$
\psi_{1} * 1 * 1 * \partial \psi^{2} / \partial n=-\psi_{1} * 1 * 1 * \psi_{2}(\alpha / \beta),
$$

and so it follows that the left-hand side of (4.1) vanishes. Thus

$$
\begin{aligned}
0 & =\int\left(\psi_{1} * 1 * 1 * \nabla^{2} \psi_{2}-\psi_{2} * 1 * 1 * \nabla^{2} \psi_{1}\right) d \tau \\
& =\frac{1}{c^{2}} \int\left[\psi_{1} *\left(\psi_{2}+p_{2}\right)-\psi_{2} *\left(\psi_{1}+p_{1}\right)\right] d \sigma
\end{aligned}
$$

whence

$$
\int \psi_{1} * p_{2} d \tau=\int \psi_{2} * p_{1} d \tau,
$$

which is the reciprocity theorem connecting the "disturbance" and "source".

The problem of an infinite space may be treated as follows. Suppose that the boundary is a hypersphere of radius $R$, centered at the origin, $R$ being large. An element of the boundary is thus $O\left(R^{n-1}\right)$. Suppose that, on $|\mathbf{x}|=R$, instead of the boundary condition (2.2), $\psi$ obeys the order condition

$$
\psi=o\left(R^{-(n / 2)+1}\right)
$$

and the generalized Sommerfeld radiation condition

$$
\chi=\frac{1}{c} \frac{\partial \psi}{\partial t}+\frac{\partial \psi}{\partial R}=o\left(R^{-n / 2}\right) .
$$

The integral over the boundary in (4.1) is

$$
\begin{aligned}
\int_{B}\left(\psi_{1} * 1 * 1 * \frac{\partial \psi_{2}}{\partial n}-\right. & \left.\psi_{2} * 1 * 1 * \frac{\partial \psi_{1}}{\partial n}\right) d \sigma \\
= & \int_{|\mathbf{x}|-R}\left(\psi_{1} * 1 * 1 * \frac{\partial \psi_{2}}{\partial R}-\psi_{1} * 1 * 1 * \frac{\partial \psi_{1}}{\partial R}\right) d \sigma \\
= & \int_{|\mathbf{x}|-R}\left(\psi_{1} * 1 * 1 * \chi_{2}-\psi_{2} * 1 * 1 * \chi_{1}\right) d \sigma \\
& +\frac{1}{c} \int_{|\mathbf{x}| * R}\left(\psi_{2} * 1 * 1 * \frac{\partial \psi_{1}}{\partial t}-\psi_{2} * 1 * 1 * \frac{\partial \psi_{2}}{\partial t}\right) d \sigma
\end{aligned}
$$

Using (2.4) it follows that

$$
1 * \frac{\partial \psi_{1}}{\partial t}=\psi_{1} \quad \text { and } \quad 1 * \frac{\partial \psi_{2}}{\partial t}=\psi_{2}
$$

and so

$$
\psi_{2} * 1 * 1 * \frac{\partial \psi_{1}}{\partial t}=\psi_{1} * 1 * 1 * \frac{\partial \psi_{2}}{\partial t},
$$

and the boundary integral becomes

$$
\int_{|\mathbf{x}|-R}\left(\psi_{1} * 1 * 1 * \chi_{2}-\psi_{2} * 1 * 1 * \chi_{1}\right) d \sigma .
$$


Now

$$
\psi \chi d \sigma=o\left(R^{-n / 2}\right) o\left(R^{-(n / 2)+1}\right) O\left(R^{n-1}\right)=o(1),
$$

and so (4.3a) and (4.3b) form a set of sufficient conditions for the left-hand side of (4.1) to vanish when the boundary is at infinity. If part of the boundary is finite and part infinite, the extension is obvious.

A particular case of (4.2) is associated with the Green's function. Let $G\left(\mathbf{x}, \mathbf{x}^{\prime} ; t\right)$ be the solution of the differential equation

$$
\nabla^{2} G-\frac{1}{c^{2}} \frac{\partial^{2} G}{\partial t^{2}}=\delta\left(\mathbf{x}-\mathbf{x}^{\prime}\right) \delta(t)
$$

which is associated with the initial conditions

$$
G=0, \quad \partial G / \partial t=0 \quad \text { at } t=0 .
$$

Suppose that $G_{0}$ is the solution of this equation under the boundary condition (4.3), this being actually the radiation condition here. Suppose that $G=G_{0}+G^{*}$ is the solution of this equation under the boundary condition

$$
\alpha G+\beta \partial G / \partial n=0 .
$$

$G^{*}$ may be determined as follows.

From Eq. (4.8),

it follows that

$$
\nabla^{2} G_{0}+\nabla^{2} G^{*}-\frac{1}{c^{2}} \frac{\partial^{2} G_{0}}{\partial t^{2}}-\frac{1}{c^{2}} \frac{\partial^{2} G_{0}}{\partial t^{2}}=\delta\left(\mathbf{x}-\mathbf{x}^{\prime}\right) \delta(t) ;
$$

$$
\nabla^{2} G^{*}-\frac{1}{c^{2}} \frac{\partial^{2} G^{*}}{\partial t^{2}}=0
$$

The initial conditions for $G^{*}$ will clearly be given by

$$
G^{*}=0, \quad \frac{\partial G^{*}}{\partial t}=0, \quad t=0,
$$

and the boundary condition by

$$
\alpha G^{*}+\beta \frac{\partial G^{*}}{\partial h}=-\alpha G_{0}-\beta \frac{\partial G_{0}}{\partial n} .
$$

$G^{*}$ is thus defined by Eqs. (4.11a), (4.11b) and (4.11c) and can be solved in the usual way. This problem has been discussed by Jones [5].

The "source" $p_{G}$ associated with the "excitation" $G$ is

$$
c^{2}\left[1 * 1 * \delta\left(\mathbf{x}-\mathbf{x}^{\prime}\right) \delta(t)\right]=c^{2} \delta\left(\mathbf{x}-\mathbf{x}^{\prime}\right) 1 * 1 \quad\left(=c^{2} \delta\left(\mathbf{x}-\mathbf{x}^{\prime}\right) t .\right) .
$$

(4.2) takes the form

$$
\int\left(\psi * p_{G}\right) d \tau=\int\left(G * p_{G}\right) d \tau
$$

Writing the above equation in full,

$$
\int c^{2} \delta\left(\mathbf{x}-\mathbf{x}^{\prime}\right) \psi(\mathbf{x}, t) * 1 * 1 d \tau=\int G\left(\mathbf{x}, \mathbf{x}^{\prime}, t\right) * p(\mathbf{x}, t) .
$$


Using the properties of the delta function, this takes the form

$$
c^{2} 1 * 1 * \psi\left(\mathbf{x}^{\prime}, t\right)=\int G\left(\mathbf{x}, \mathbf{x}^{\prime}, t\right) p(\mathbf{x}, t)
$$

and using (2.4) twice, it follows that

$$
\psi\left(\mathbf{x}^{\prime}, t\right)=\frac{1}{c^{2}} \frac{\partial^{2}}{\partial t^{2}} \int G\left(\mathbf{x}, \mathbf{x}^{\prime}, t\right) p(\mathbf{x}, t) d \tau .
$$

One particular "excitation" which is of interest is that which is associated with the "source" $\delta\left(\mathbf{x}-\mathbf{x}_{0}\right) \delta\left(t-t_{0}\right) t_{0}>0$. This is given by

$$
\begin{aligned}
\psi_{1}\left(\mathbf{x}^{\prime}, t\right) & =\frac{1}{c^{2}} \frac{\partial^{2}}{\partial t^{2}} \int G\left(\mathbf{x}, \mathbf{x}^{\prime}, t\right) \delta\left(t-t_{0}\right) \delta\left(\mathbf{x}-\mathbf{x}_{0}\right) d \tau \\
& =\frac{1}{c^{2}} \frac{\partial^{2}}{\partial t^{2}} G\left(\mathbf{x}_{0}, \mathbf{x}^{\prime}, t-t_{0}\right) .
\end{aligned}
$$

These formulae make it possible to determine an excitation directly in terms of the quantities $f(\mathbf{x}, t), g(\mathbf{x}), \phi_{1}(\mathbf{x})$, and $\phi_{0}(\mathbf{x})$.

5. Uniqueness. It is necessary to determine the conditions under which the solution of (2.1) is unique. Uniqueness exists if there is no nontrivial solution of the system defined by the differential equation

$$
\nabla^{2} \phi-\frac{1}{c^{2}} \frac{\partial^{2} \phi}{\partial t^{2}}=0
$$

together with the initial conditions

$$
\phi=0 \text { and } \frac{\partial \phi}{\partial t}=0 \text {, at } t=0
$$

and the appropriate boundary condition: for finite boundaries

$$
\alpha \phi+\beta \frac{\partial \phi}{\partial n}=0
$$

and for infinite boundaries

$$
\phi, \frac{\partial \phi}{\partial t}=o\left(R^{-n / 2+1}\right), \quad \chi=\frac{\partial \phi}{\partial R}+\frac{1}{c} \frac{\partial R}{\partial t}=o\left(R^{-n / 2}\right) .
$$

Consider the quantity

$$
E=\frac{1}{2} \int\left[(\nabla \phi)^{2}+\frac{1}{c^{2}}\left(\frac{\partial \phi}{\partial t}\right)^{2}\right] d \tau .
$$

$E$ is intrinsically either positive or zero. Initially $\phi$ is zero and hence $\nabla \phi$ is zero. Also $\partial \phi / \partial t$ is zero. Thus initially $E$ is zero.

$$
\begin{aligned}
\frac{\partial E}{\partial t} & =\int\left[(\nabla \phi) \cdot \nabla\left(\frac{\partial \phi}{\partial t}\right)+\frac{1}{c^{2}} \frac{\partial \phi}{\partial t} \frac{\partial^{2} \phi}{\partial t^{2}}\right] d \tau \\
& =\int\left[(\nabla \phi) \cdot \nabla \frac{\partial \phi}{\partial t}+\frac{\partial \phi}{\partial t} \nabla^{2} \phi\right] d \tau \\
& =\int \nabla \cdot\left(\frac{\partial \phi}{\partial t} \nabla \phi\right) d \tau=\int_{B} d \sigma \frac{\partial \phi}{\partial t} \frac{\partial \phi}{\partial n} .
\end{aligned}
$$


Suppose now that $B$ is finite. If either $\phi$ or $\partial \phi / \partial n$ vanishes on $B$, the right-hand side of (5.5) is zero and so $\partial E / \partial t$ vanishes, $E$ remains zero and there is uniqueness. If (5.3a) holds

$$
\frac{\partial E}{\partial t}=-\int_{B} d \sigma \frac{\partial \phi}{\partial t} \frac{\alpha}{\beta} \phi=-\frac{\partial}{\partial t} \int d \sigma \frac{\alpha}{\beta} \frac{\phi^{2}}{2}
$$

and

$$
\frac{\partial}{\partial t}\left[E+\frac{1}{2} \int \frac{\alpha}{\beta} \phi^{2} d \sigma\right]=0 .
$$

If $\alpha / \beta>0, E^{1}=E+\frac{1}{2} \int(\alpha / \beta) \phi^{2} d \sigma$ is intrinsically positive or zero, it is initially zero and $\partial E^{1} / \partial t=0$. Thus $E^{1}$ is always zero, $\phi$ is zero and there is uniqueness. If, however, $\alpha / \beta$ is not always positive, this does not hold, and uniqueness may not exist.

If now the boundary is infinite,

$$
\frac{\partial E}{\partial t}=\int_{B} d \sigma \frac{\partial \phi}{\partial t} \frac{\partial \phi}{\partial n}=\int_{|\mathbf{x}|=R} d \sigma \frac{\partial \phi}{\partial t} \frac{\partial \phi}{\partial R}=\int_{|\mathbf{x}|=R} d \sigma\left[\frac{\partial \phi}{\partial t} \chi\right]-\frac{1}{c} \int_{|\mathbf{x}|=R} d \sigma\left(\frac{\partial \phi}{\partial t}\right)^{2} .
$$

The order of magnitude of the first integral on the right-hand side of (5.7) is given by $O\left(R^{n-1}\right) o\left(R^{-n / 2+1}\right) o\left(R^{-n / 2}\right)=o(1)$ and so vanishes as $R$ tends to infinity.

The order of magnitude of the second integral on the right-hand side of (5.7) is

$$
O\left(R^{n-1}\right) o\left(R^{-n / 2+1}\right) o\left(R^{-n / 2+1}\right) .
$$

This may be expressed as $O(1) o(R)$, but cannot be reduced further. This does not matter however, as the right-hand side of (5.7) is clearly negative or zero, and $\partial E / \partial t \leq 0$. From this, it follows again that $\phi$ is identically zero and there is uniqueness.

Appendix. The case of $n=1$ can also be treated, but certain modifications are necessary. The region under consideration becomes a strip of the real axis $x_{1}<x<x_{2}$, the boundary $B$ becomes the two points $x_{1}, x_{2}$ and the boundary condition becomes

$$
\begin{array}{ll}
\alpha_{1} \phi+\beta_{1} \frac{d \phi}{d x}=g_{1} & x=x_{1}, \\
\alpha_{2} \phi-\beta_{2} \frac{d \phi}{d x}=g_{2} & x=x_{2} ; \\
\int f d \tau \text { becomes } & \int_{x_{1}}^{x_{2}} f(x) d x
\end{array}
$$

and

$$
\begin{aligned}
& \int_{B} f d \sigma=f\left(x_{2}\right)-f\left(x_{1}\right), \\
& \int_{B_{1}} f d \sigma=f\left(x_{2}\right)-f\left(x_{1}\right) .
\end{aligned}
$$

In (A.4), if $\beta_{1}$ is zero, $f\left(x_{1}\right)$ is omitted and, if $\beta_{2}$ is zero, $f\left(x_{2}\right)$ is omitted.

T. Gren's function which satisfies the radiation condition is

$$
G_{0}(x, t)=-\frac{c}{2} H\left(1-\frac{\mid c i}{c}\right)
$$

where $H(t)$ is the unit function. 
Acknowledgement. I am grateful to the refreee for some comments on the first version of this paper.

\section{REFERENCES}

[1] M. E. Gurtin, Variational principle for the time-dependent Schrödinger equation, J. Mathematical Phys. 6, 1506-1507 (1965)

[2] M. E. Gurtin, Variational principles for linear initial value problems, Quart. Appl. Math. 22, 252256 (1964)

[3] L. Ainola, The variation principle for the Schrödinger equation, Eesti NSV Tead. Akad. Toimetised Füüs-Mat. 16, 139-142 (1967)

[4] D. S. Jones, Generalized functions, McGraw-Hill, New York, 1966, p. 156

[5] D. S. Jones, The theory of electromagnetism, Pergamon Press, New York, 1964, pp. 672-676 